\title{
COMPARAÇÃO ENTRE MÉTODOS FUZZY NA LOCALIZAÇÃO DE UMA BASE NAVAL HIPOTÉTICA
}

\author{
Leonardo Antonio Monteiro Pessôa \\ Centro de Análises de Sistemas Navais-CASNAV/COPPE-UFRJ \\ lampessoa@terra.com.br \\ Marcos Pereira Estellita Lins \\ COPPE-UFRJ \\ estellita@pep.ufrj.br \\ Carlos Alberto Nunes Cosenza \\ COPPE-UFRJ \\ cosenza@pep.ufrj.br \\ Helder Gomes Costa \\ Universidade Federal Fluminense - UFF \\ hgc@vm.uff.br
}

\begin{abstract}
Resumo
As preocupações com a região amazônica brasileira ditam boa parte do tom da Estratégia Nacional de Defesa, inclusive determinando estudos e preparativos para instalação de uma base naval nas proximidades da foz do rio Amazonas. Muitas vezes, entretanto, os tomadores de decisão lidam com critérios subjetivos, imprecisão e vagueza inerentes às caracterizações das alternativas. Reconhece-se que a lógica difusa (fuzzy) lida justamente para o tratamento desta natureza. Imaginando este cenário, o presente trabalho procura comparar diferentes metodologias de localização $F u z z y$ na seleção de local para uma hipotética base naval. Para síntese do quadro metodológico, este trabalho utiliza técnicas desenvolvidas por Cosenza (Lima, Cosenza, and das Neves 2009), (Cosenza et al. 2006), Liang \& Wang (Liang and Wang 1991) e Baas \& Kwakernaak, (Baas \& Kwakernaak, 1977), as quais utilizam lógica fuzzy como base. Este trabalho não pretende servir como base para a futura decisão da Marinha do Brasil, mas sim apresentar exemplo de utilização didática, divulgando molduras para o auxílio a esta decisão e, possivelmente, para outros problemas relacionados à esfera militar.
\end{abstract}

Palavras-Chave: Multicritério; Fuzzy; Localização; Base Naval

\section{Abstract}

The Amazon Region is presented as one of the major concerns in the Brazilian National Defence Strategy, guiding studies and arrangements for the development of a Naval Base near of the Amazon river mouth. However, decision makers deal with qualitative criteria, with inherent imprecision and vagueness regarding the alternatives and fuzzy logic 
deals specifically with this nature. The present paper compares different location selection methodologies for a hypothetical Naval Base complying with the aforementioned scenario. Fuzzy Logic based methodologies created by Baas \& Kwakernaak (Baas \& Kwakernaak, 1977), Liang \& Wang (Liang and Wang 1991), and Cosenza (Lima, Cosenza, and das Neves 2009) (Cosenza et al. 2006), are considered. The aim of this work is to present, as a didactic example, different frameworks that could aid that decision and, possibly, other problems related to the military, but this paper is not intended to guide the actual decision of the Brazilian Navy.

Keywords: MCDA; Fuzzy; Location; Naval Base

\section{INTRODUÇÃO}

A seleção de localizações é considerada particularmente importante para organizações governamentais e industriais, para maximização do uso de recursos e minimização do custo total (Liang and Wang 1991). A Pesquisa Operacional contribui para a resolução deste problema com o arcabouço metodológico, particularmente relacionado à análise multicritério à decisão.

Muitas vezes, entretanto, os tomadores de decisão lidam com critérios subjetivos com imprecisão e vagueza inerentes às caracterizações das alternativas. Reconhece-se que a lógica difusa (fuzzy) teve sua criação justamente para o tratamento desta natureza.

O objetivo do presente trabalho é o de comparar, quanto à diferença de dominância, quanto a facilidade de implementação e destacando aspectos de flexibilidade, três diferentes técnicas baseadas em lógica fuzzy, desenvolvidas por Cosenza (Lima, Cosenza, and das Neves 2009) (Cosenza et al. 2006), por Liang \& Wang (Liang and Wang 1991) e por Baas \& Kwakernaak (Baas and Kwakernaak 1977). Utiliza-se para esta finalidade o problema de localização de uma base naval hipotética. Este exemplo é contextualizado propositalmente para alinhar-se a aspecto estratégico real, no tocante à região amazônica.

As preocupações com a região amazônica brasileira ditam boa parte do tom da Estratégia Nacional de Defesa (Brasil 2008), documento promulgado pelo Decreto $\mathrm{n}^{\mathrm{o}}$ 6.703.

"A Marinha iniciará os estudos e preparativos para estabelecer, em lugar próprio, o mais próximo possível da foz do rio Amazonas, uma base naval de uso múltiplo, comparável, na abrangência e na densidade de seus meios, à Base Naval do Rio de Janeiro."

Este trabalho não pretende servir como base para a futura decisão da Marinha do Brasil, mas sim apresentar exemplo de utilização didática, divulgando molduras para o auxílio a esta decisão, e possivelmente para outros problemas relacionados à esfera militar.

A fim de explicitar o desenvolvimento do trabalho, na seção 2 é apresentado o referencial teórico. Os atributos (critérios) considerados para avaliação e as avaliações de cada alternativa são descritos na seção 3, enquanto a seção 4 mostra os resultados obtidos. Considerações finais e conclusões do trabalho são apresentadas na seção 5. 


\section{REFERENCIAL TEÓRICO}

\subsection{LIANG - WANG}

Na metodologia descrita em Liang e Wang (1991) Os atributos relativos ao local podem ser divididos em dois grupos: Atributos objetivos (definidos em termos financeiros); e atributos de definição qualitativa.

O método utiliza uma medida chamada fuzzy suitability index responsável por quantificar a ambiguidade envolvida na avaliação da propriedade (aceitabilidade) das alternativas e da importância dos critérios.

São utilizados números fuzzy trapezoidais os quais podem ser definidos da seguinte maneira: Seja A um numero trapezoidal em R, sua função de pertinência é:

$$
f_{A}(x)=\left\{\begin{array}{c}
\frac{x-c}{a-c}, \text { se } c \leq x \leq a \\
1, \text { se } a \leq x \leq b \\
\frac{x-b}{d-b}, \text { se } b \leq x \leq d \\
0, \text { demais casos }
\end{array}\right.
$$

Onde $c \leq a \leq b \leq d$, podendo ser representado como (c,a,b,d)

Este método contempla a possibilidade de utilização de $\mathrm{n}$ decisores $\left(D_{1}, \ldots, D_{n}\right)$, responsáveis pela avaliação de $\mathrm{k}$ critérios $\left(C_{1}, \ldots, C_{k}\right)$, quanto sua importância relativa, e também pela avaliação de m alternativas $\left(A_{1}, \ldots, A_{m}\right)$, acerca destes critérios.

Seja $S_{i t j}$ a avaliação definida pelo avaliador $D_{j}$ para o critério $C_{t}$ em relação à alternativa $A_{i}$. $S_{i t}$ Representa a agregação das avaliações dos $\mathrm{j}$ decisores, da alternativa $A_{i}$ para o critério $C_{t}$. $W_{t}$ é o peso relativo ao critério $C_{t}$

$F_{i}$ é a avaliação final da alternativa $A_{i}$, obtida pela agregação de $S_{i t}$ e $W_{t}$ para todos os critérios, obtendo o ranqueamento final de alternativas por ordenação de todas as avaliações finais. As variáveis linguísticas da importância dos critérios apresentam a seguinte correspondência para números fuzzy trapezoidais: Very Low - VL $(0 ; 0 ; 0 ; 0,3)$; Low - L $(0 ; 0,3 ; 0,3 ; 0,5)$; Medium - M $(0,2 ; 0,3 ; 0,3 ; 0,5)$; High - H $(0,5 ; 0,7 ; 0,7 ; 1)$; e Very High - VH $(0,7 ; 1 ; 1 ; 1)$.

$\mathrm{Na}$ avaliação de alternativas utilizam-se as variáveis: Very Poor - VP $(0 ; 0 ; 0 ; 0,2)$; Between Very Poor \& Poor - BVP\&P $(0 ; 0 ; 0,2 ; 0,4)$; Poor - P $(0 ; 0,2 ; 0,2 ; 0,4)$; Between Very Poor \& Fair - BP\&F $(0 ; 0,2 ; 0,5 ; 0,7)$; Fair - F $(0,3 ; 0,5 ; 0,5 ; 0,7)$; Between Fair \& Good - BF\&G $(0,3 ; 0,5 ; 0,8 ; 1)$; Good - G $(0,6 ; 0,8 ; 0,8 ; 1)$; Between Good \& Very Good - BG\&VG $(0,6 ; 0,8 ; 1 ; 1) ;$ e Very Good - VG $(0,8 ; 1 ; 1 ; 1)$

Como há possibilidade de utilização de critérios objetivos e subjetivos, o método converte os custos dos critérios objetivos (monetários) em índice adimensional, no qual as alternativas com mínimo custo recebem a melhor avaliação.

Sejam $T_{i}, i=1,2, \ldots, m$ os critérios objetivos, a representação do custo total de $A_{i}$ é $R T_{i}=\left\{T_{i} \otimes\left[T_{1}^{-1} \oplus T_{2}^{-1} \oplus \ldots \oplus T_{m}^{-1}\right]\right\}^{-1}$

A agregação das avaliações utiliza um operador de média, utilizando as seguintes 
definições:

$S_{i t j}=\left(q_{i t j}, o_{i t j}, p_{i t j}, r_{i t j}\right)$ representa o número fuzzy trapezoidal correspondente a avaliação de $A_{i}$ pelo decisor j acerca do critério t.

$S_{\text {it }}$

$$
\begin{aligned}
& =\left\{\begin{array}{c}
\frac{1}{n} \otimes\left(S_{i t 1} \oplus S_{i t 2} \oplus \ldots \oplus S_{i t j}\right), \text { onde } t=(1,2, \ldots k-1) \text { compreende os critérios subjetivos } \\
\left(q_{i k}, o_{i k}, p_{i k}, r_{i k}\right), \quad t=k \text { referente ao critério objetivo }
\end{array}\right. \\
& S_{i t}=\left(q_{i t}, o_{i t}, p_{i t}, r_{i t}\right)
\end{aligned}
$$

Onde $q_{i t}=\sum_{i}^{n} \frac{q_{i t j}}{n}, o_{i t}=\sum_{i}^{n} \frac{o_{i t j}}{n}, q_{i t}=\sum_{i}^{n} \frac{p_{i t j}}{n}, q_{i t}=\sum_{i}^{n} \frac{r_{i t j}}{n}$

$$
W_{t}=\frac{1}{n} \otimes\left(W_{t 1} \oplus W_{t 2} \oplus \ldots \oplus W_{t n}\right), W_{t}=\left(c_{t}, a_{t}, b_{t}, d_{t}\right), c_{t}=\sum_{i}^{n} \frac{c_{t j}}{n}, a_{t}=\sum_{i}^{n} \frac{a_{t j}}{n}, b_{t}=\sum_{i}^{n} \frac{b_{t j}}{n}, d_{t}=\sum_{i}^{n} \frac{d_{t j}}{n}
$$

É realizada a agregação de $S_{i t}$ e $W_{t}$ pela média de seus produtos sobre todos os critérios, obtendo o fuzzy suitability index $F_{i}$

$$
F_{i}=\frac{1}{k} \otimes\left\{\left[S_{i 1} \otimes W_{1}\right] \oplus\left[S_{i 2} \otimes W_{2}\right] \oplus \ldots \oplus\left[S_{i k} \otimes W_{k}\right]\right\}
$$

Este será um número fuzzy com função de pertinência:

Onde:

$$
f_{F_{i}}=\left\{\begin{array}{c}
-H_{i 1}+\left[{H_{i 1}}^{2}+\frac{\left(x-Y_{i}\right)}{T_{i 1}}\right]^{1 / 2}, \text { para } Y_{i} \leq x \leq Q_{i} \\
1, \text { para } Q_{i} \leq x \leq R_{i} \\
H_{i 2}-\left[{H_{i 2}}^{2}+\frac{\left(x-Z_{i}\right)}{U_{i 1}}\right]^{\frac{1}{2}}, \text { para } R_{i} \leq x \leq Q_{i} \\
0, \text { demais casos }
\end{array}\right.
$$

$T_{i 1}=\sum_{t=1}^{k} \frac{\left(o_{i t-} q_{i t}\right)\left(a_{t-} c_{t}\right)}{k} \quad T_{i 2}=\sum_{t=1}^{k} \frac{\left[q_{i t}\left(a_{t-} c_{t}\right)+c_{t}\left(o_{i t-} q_{i t}\right)\right]}{k}$

$U_{i 1}=\sum_{t=1}^{k} \frac{\left(r_{i t-} p_{i t}\right)\left(d_{t-} b_{t}\right)}{k} T_{i 2}=\sum_{t=1}^{k} \frac{\left.d_{t}\left(p_{i t-} r_{i t}\right)+r_{i t}\left(b_{i-} d_{i}\right)\right]}{k}$

$H_{i 1}=\frac{T_{i 2}}{2 T_{i 1}} H_{i 2}=\frac{-U_{i 2}}{2 U_{i 1}}$

De maneira simplificada representa-se o Fuzzy suitability index como um número trapezoidal $F_{i} \cong\left(Y_{i}, Q_{i}, R_{i}, Z_{i}\right)$.

Para a ordenação é usado o método de Chen, considerando para isso um conjunto maximizante $M=\left\{x, f_{M}(x) \mid x \in R\right\}$ e um minimizante $G=\left\{x, f_{g}(x) \mid x \in R\right\}$, com funções de pertinência:

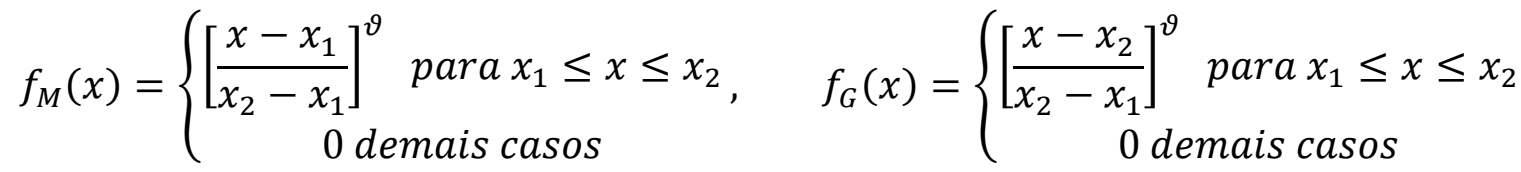

$\vartheta$ é um parâmetro para ajuste da aplicação quanto ao incremento da função de preferência do decisor, sendo estritamente positivo. Onde: 
$E_{i}=\left\{x, f_{F i}(x)>0\right\}, E=\bigcup_{i=1}^{m} E_{i} x_{1}=\inf E, x_{2}=\sup E$

Definem-se as utilidades maximizante, minimizante e total:

$$
\begin{gathered}
U_{M}\left(F_{i}\right)=\sup _{x}\left(f_{F i}(x) \wedge f_{M}(x)\right), U_{G}\left(F_{i}\right)=\sup _{x}\left(f_{F i}(x) \wedge f_{G}(x)\right) \\
U_{T}\left(F_{i}\right)=\frac{\left[U_{M}\left(F_{i}\right)+1-U_{G}\left(F_{i}\right)\right]}{2}
\end{gathered}
$$

Destaca-se que em Liang and Wang (1991) é apresentada uma simplificação para $U_{T}$ por meio da aproximação do número trapezoidal $\left(Y_{i}, Q_{i}, R_{i}, Z_{i}\right)$ :

$$
U_{T}(F i) \cong \frac{\left[\frac{Z_{i}-x_{1}}{\left(x_{2}-x_{1}\right)-\left(R_{i}-Z_{i}\right)}+1-\frac{\left(x_{2}-Y_{i}\right)}{\left(x_{2}-x_{1}\right)+\left(Q_{i}-Y_{i}\right)}\right]}{2}
$$

$R_{j}$.

Considera-se que $F_{i}>F_{j}$ se $U_{T}\left(F_{i}\right)>U_{T}\left(F_{j}\right)$ ou se $F_{i}=F_{j}$, mas $Q_{i}+R_{i}>Q_{j}+$

\subsection{BAAS-KWAKERNAAK}

O modelo Baas-Kwakernaak, considerado como clássico e referência para outros métodos fuzzy (Khamaran 2008), é um modelo de avaliação média ponderada que utiliza lógica fuzzy para representação da incerteza ou de imprecisão das avaliações, tanto dos pesos aplicados aos critérios, quanto das avaliações de cada alternativa em relação aos critérios.

Dadas $A_{i}$ alternativas $i=\{1, \ldots, m\}$

Avaliadas por $c_{j}$ critérios $j=\{1, \ldots, n\}$, onde cada critério é considerado relevante para todas as alternativas, por uma importância relativa denominada $w_{j}$.

As alternativas são avaliadas em relação a cada critério, por uma função de pertinência $\mu_{R i j}\left(r_{i j}\right)$ E cada critério tem sua importância relativa pela função de pertinência $\mu_{w j}\left(w_{j}\right)$, onde $r_{i j}$ e $w_{j}$ assumem valores na linha positiva em R, em um espaço finito.

As funções de pertinência assumem valores em $[0,1]$, além de assumirem ao menos uma vez o valor 1 como argumento.

Originalmente são descritos os seguintes números fuzzy, aqui descritos como trapezoidais, para representar os pesos dos critérios: Very Important - VI $(0,8 ; 1 ; 1 ; 1)$; Moderately Important - MI (0,3;0,5;0,5;0,7); e Relatively Unimportant - RUI (0;0,2;0,2;0,4).

Para avaliação das alternativas também são descritos os seguintes níveis: Poor - $\mathrm{P}$ $(0 ; 0,2 ; 0,2 ; 0,4)$; Fair - F $(0,4 ; 0,6 ; 0,6 ; 0,8)$; Fair to Good - FG $(0,4 ; 0,6 ; 0,8 ; 1) ;$ Good - G $(0,6 ; 0,8 ; 0,8 ; 1)$; Very Good - VG $(0,8 ; 1 ; 1 ; 1)$

Para a determinação da avaliação da alternativa $A_{i}$ é produzido o mapeamento de $R^{2 n}$ em $R$ dado pela função $g$.

$$
g(z)=\frac{\sum_{j=1}^{n} w_{j} r_{j}}{\sum_{j=1}^{n} w_{j}}
$$

Onde $\mu_{z i}=\left[\Lambda_{j=1}^{n} \mu\left(w_{j}\right)\right] \wedge\left[\bigwedge_{k=1}^{n} \mu_{R i k}\left(r_{k}\right)\right]$, e $z=\left(w_{1}, \ldots, w_{n}, r_{1}, \ldots, r_{n}\right)$

Então o conjunto fuzzy $z=\left(R^{2 n}, \mu_{z i}\right)$ induz o conjunto fuzzy $\bar{R}_{i}=\left(R, \mu_{\bar{R}}\right)$, que caracteriza a avaliação final da alternativa $A_{i}$ de um modo fuzzy, por meio da função de 
pertinência:

$$
\mu_{\bar{R} i}(\bar{r})=\sup _{z: g(z)=\bar{r}} \mu_{z i}(z), \quad r \in R
$$

Para comparação entre as avaliações de cada alternativa definem-se:

$$
\mu_{I \mid R}\left(i \mid \overline{r_{1}}, \ldots, \overline{r_{m}}\right)=\left\{\begin{array}{c}
1 \text { se } r_{i} \geq r_{j}, \forall j \in I \\
0, \text { caso contrário }
\end{array}, \text { e } \mu_{\bar{R}}\left(\overline{r_{1}}, \ldots, \overline{r_{m}}\right)=\bigwedge_{i=1}^{m} \mu_{\bar{R} i}\left(\overline{r_{l}}\right)\right.
$$

Induzindo o conjunto fuzzy $\left(I, \mu_{I}\right)$, com função de pertinência:

$$
\mu_{I}(i)=\sup _{\overline{r_{1}} \ldots \overline{r_{m}}} \mu_{I \mid R}\left(i \mid \overline{r_{1}}, \ldots, \overline{r_{m}}\right) \wedge \mu_{\bar{R}}\left(\overline{r_{1}}, \ldots, \overline{r_{m}}\right)
$$

Que proverá de maneira parcial o grau de preferência de uma alternativa em relação ao conjunto. A avaliação final de uma alternativa é comparada em relação às demais utilizando: $p_{i}=r_{i}-\frac{1}{m-1} \sum_{\substack{i=1 \\ i \neq j}}^{m} \bar{r}_{j}$

Que induz o conjunto fuzzy $P i=\left(R, \mu_{P i}\right)$, com função de pertinência:

$$
\mu_{P i}(p)=\sup \underset{h=\overline{\left(r_{1}, \ldots, \ldots, \overline{r_{m}}\right)}=p}{\overline{r_{2}}} \mu_{\bar{R}}\left(\overline{r_{1}}, \ldots, \overline{r_{m}}\right), p \in \mathrm{R}
$$

A qual completa a avaliação, mostrando a extensão de superioridade de uma alternativa sobre as demais.

\subsection{COPPE - COSENZA}

O modelo Coppe-Cosenza tem uma estrutura particular no tratamento do problema permitindo a análise de diversos projetos simultaneamente.

Os conceitos primordiais foram extraídos de (Cosenza 2009), no qual pode ser consultada uma análise matemática mais profunda. Igualmente, exemplos prévios de utilização do modelo também podem ser encontrados em (Lima et al. 2009), (Cosenza et al. 2006) Sejam h projetos a serem analisados, com demandas sintetizadas em n critérios, dentro de um conjunto de $m$ alternativas, teremos:

$A=\left(a_{i j}\right)_{h X n}$ a matriz que representa as demandas dos h projetos em $\mathrm{n}$ critérios;

$B=\left(b_{j k}\right)_{n X m}$ a matriz que representa a oferta das $\mathrm{m}$ alternativas locacionais em relação aos n critérios analisados; e

$F=\left\{f_{i} \mid 1, \ldots, n\right\}$ representa o conjunto dos $n$ critérios (fatores) analisados.

$\tilde{A}=\left\{\left(f, \mu_{\tilde{A}}(f) \quad \mid f \in F\right\}\right.$ é o conjunto fuzzy de pares ordenados em $\mathrm{f}$, sendo a representação fuzzy da matriz $\mathrm{A}$, onde $\mu_{\tilde{A}}(f)$ é o grau de importância do fator, tratado nas variáveis linguísticas: Crítico; Condicionante; Pouco Condicionante; e Irrelevante.

De maneira análoga alternativa.

$\tilde{B}=\left\{\left(f, \mu_{\tilde{B}}(f) \mid f \in F\right\} \quad\right.$ representa o grau de atendimento dos fatores pela

Neste aspecto há um diferencial quanto à ausência de oferta de um fator considerado. Como o modelo pode ser utilizado para avaliação simultânea de vários projetos, há a possibilidade que determinados fatores críticos ou condicionantes para um projeto sejam pouco condicionantes, ou irrelevantes para outros. Para melhor tratamento desta realidade são descritas classificações diferenciadas para representação de ausência:

Vazio: Representando condições para desenvolvimento futuro de oferta do fator 
considerado - $\varnothing$

Nulidade: significando a ausência determinada do fator, e que não há possibilidades de obtenção do mesmo, podendo representar, por exemplo, recursos naturais - (0). Inexistência: significando a inexistência do fator, como por exemplo, matérias primas - $\nexists$;

Havendo o fator, o mesmo será descrito nas variáveis linguísticas: Superior - S; Bom - B; Regular - R; e Fraco - F.

É definida uma matriz consolidando os requisitos de demanda, representados por coeficientes fuzzy, para todos os projetos. Onde cada elemento $a_{i j}$ representa o nível requerido do fator (critério) $\boldsymbol{f}_{j}$ pelo projeto $\widetilde{\boldsymbol{A}_{l}}$

E outra matriz que consolida coeficientes fuzzy relativos à oferta dos fatores por cada alternativa. Sendo $b_{j k}$ o nível do fator $\boldsymbol{f}_{\boldsymbol{j}}$ disponibilizado pela alternativa $\widetilde{\boldsymbol{B}_{\boldsymbol{k}}}$.

É definido $C=A \otimes B=\left(c_{i k}\right)_{h X m}$, onde $c_{i k}$ é o coeficiente de avaliação da alternativa $\mathrm{k}$ em relação ao projeto $\mathrm{m}$. No modelo Coppe-Cosenza, o operador $\otimes$ pode assumir diferentes regras operacionais e permitir a utilização de modificadores clássicos de dilatação ou contração, os quais podem ser consultados nas referências.

Para o exemplo apresentado posteriormente, será utilizada a regra operacional de matriz triangular, conforme descreve a tabela 1 :

Tabela 1 - Operação de Agregação Cosenza

\begin{tabular}{cccccllll}
\hline $\boldsymbol{a}_{\boldsymbol{i} \boldsymbol{j}} \otimes \boldsymbol{b}_{\boldsymbol{j} \boldsymbol{k}}$ & $\nexists$ & $\{\mathbf{0}\}$ & $\varnothing$ & Fraco & Regular & Bom & Superior \\
\hline Irrelevante & 0 & 0 & 0 & 1 & $1+1 / \mathrm{n}$ & $1+2 / \mathrm{n}$ & $1+3 / \mathrm{n}$ \\
Pouco Condicionante & 0 & 0 & 0 & 0 & 1 & $1+1 / \mathrm{n}$ & $1+2 / \mathrm{n}$ \\
Condicionante & 0 & 0 & 0 & 0 & 0 & 1 & $1+1 / \mathrm{n}$ \\
Crítico & 0 & 0 & 0 & 0 & 0 & 0 & 1 \\
\hline
\end{tabular}

Também, há neste modelo a possibilidade de atuação similar a veto, aspecto que não é considerado explicitamente nos outros modelos apresentados. Neste caso são descritos fatores locacionais ditos específicos, aos quais é dada a seguinte definição:

Seja $\bar{A}^{*}=\left({\overline{a^{*}}}_{i j}\right)_{m X n}$ a matriz de fatores específicos, tais quais todos sejam considerados imprescindíveis a $n^{\prime}$ projetos. Similarmente aos fatores não específicos, também haverá avaliações das alternativas em relação a estes critérios, denotado por $\bar{B}^{*}=$ $\left(\overline{b^{*}}{ }_{i j}\right)_{n \prime X m}$.

Temos $C^{*}=\widetilde{\bar{A}^{*}} \otimes \widetilde{B^{*}}$ representando os coeficientes fuzzy das avaliações dos fatores específicos.

Finalmente a agregação entre os fatores específicos e comuns é efetuada por regra operacional $\Gamma\left[\gamma_{i k}\right]$ apresentada na tabela 2 .

Tabela 2 - Agregação fatores específicos e comuns

\begin{tabular}{|c|c|c|}
\hline $\begin{array}{ll} & c_{i k} \\
c_{i k}^{*} & \\
\end{array}$ & $>0$ & $\varnothing$ \\
\hline 0 & 0 & 0 \\
\hline$\nexists$ & 0 & 0 \\
\hline$>0$ & $c_{i k}^{*}+c_{i k}$ & $c_{i k}^{*}$ \\
\hline
\end{tabular}




\section{CRITÉRIOS E ALTERNATIVAS}

Depois de apresentadas características dos modelos, torna-se necessário explicitar considerações necessárias para a comparação dos modelos neste problema.

Em primeiro lugar foi considerado apenas um tomador de decisão hipotético, haja vista ser explicitado procedimento para múltiplos decisores apenas no modelo de Liang \& Wang (Liang and Wang 1991).

Também o problema é considerado como um único projeto, adequando a comparação do Coppe-Cosenza em relação aos outros modelos. Consideramos útil ao leitor destacar a diferença quanto ao operador $\otimes$, para os modelos Liang e Wang e Coppe-Cosenza, já mencionada na seção anterior.

Consideramos somente localizações que disponham atualmente de portos importantes, com razoável proximidade da região da foz do Amazonas. Apesar de não nomear as alternativas, foram selecionadas quatro localizações as quais já possuem instalações portuárias, imaginando a instalação da base naval em suas proximidades ou, em caso de mobilização nacional, utilizando estes portos como possíveis núcleos para implantação.

Os critérios selecionados, a título de exemplificação puramente didática, são:

Portos Existentes (1) - variável qualitativa que descreve a facilidade para movimentação de meios de superfície de grande porte analisado de acordo com as características portuárias e de navegação.

Transporte Ferroviário (2) - variável qualitativa, analisando a densidade de rede ferroviária, sua ligação com as demais regiões do país.

Transporte Rodoviário (3) - variável qualitativa, analisando a densidade de rede rodoviária, sua ligação com as demais regiões do país.

Proteção Aérea (4) - variável qualitativa que descreve a proximidade de unidades da Força Aérea Brasileira, em condições de prover suporte aéreo. Neste caso, é considerada a proximidade de Bases Aéreas, mesmo não pertencentes ao estado da localidade analisada.

Proximidade Área de Operações (5) - variável qualitativa que descreve o quão rapidamente as unidades de meios de superfície, se baseadas na localidade, poderão se deslocar a região da foz do Amazonas.

Defesa de Minagem (6) - variável qualitativa que descreve a menor suscetibilidade da alternativa à realização de operações ofensivas de minagem.

Recursos Humanos (7) - variável qualitativa que descreve a adequação dos recursos humanos disponíveis no estado para o atendimento das necessidades da base naval, compreendendo todos os níveis de profissionalização e instrução.

$\mathrm{Na}$ tabela 3 constam as importâncias relativas dos critérios para cada um dos modelos mencionados:

Tabela 3 - Importâncias Critérios

\begin{tabular}{l|lll}
\hline Critérios & L\&W & B-K & C \\
\hline 1 & H & MI & Condicionante \\
2 & M & MI & Pouco Condicionante \\
3 & H & MI & Condicionante \\
\hline
\end{tabular}




\begin{tabular}{l|lll}
\hline 4 & H & MI & Condicionante \\
5 & VH & VI & Crítico \\
6 & L & RUI & Pouco Condicionante \\
7 & M & MI & Pouco Condicionante \\
\hline
\end{tabular}

\subsection{AvaliaÇão das Alternativas}

Apresentam-se na tabela 4 as avaliações das alternativas em relação aos critérios analisados, para cada uma das técnicas.

Tabela 4 - Avaliações das Alternativas

\begin{tabular}{l|lll|lll|lll|lll|}
\hline & \multicolumn{2}{|l}{ Alternativa 1 } & \multicolumn{3}{l}{ Alternativa 2 } & \multicolumn{3}{l}{ Alternativa 3 } & \multicolumn{3}{l}{ Alternativa 4 } \\
Critérios & L\&W & B\&K & C & L\&W & B\&K & C & L\&W & B\&K & C & L\&W & B\&K & C \\
\hline 1 & G & G & B & F & F & R & F & F & R & VG & VG & S \\
2 & G & G & B & F & F & R & F & F & R & VG & VG & S \\
3 & G & G & B & F & F & R & F & F & R & VG & VG & S \\
4 & VG & VG & S & BG\&VG & VG & S & G & G & B & F & F & R \\
5 & BG\&VG & VG & S & VG & VG & S & BG\&VG & G & B & F & F & R \\
6 & F & F & R & VG & VG & S & BG\&VG & G & B & G & G & B \\
7 & BG\&VG & VG & S & BF\&G & F & R & F & F & R & BG\&VG & VG & S \\
\hline
\end{tabular}

\section{RESULTADOS}

\subsection{FUZZY INDEX L\&W}

Utilizando a metodologia de Liang \& Wang, obtemos os seguintes Fuzzy Indexes para as Alternativas apresentados na tabela 5.

Tabela 5 - Fuzzy Indexes

\begin{tabular}{l|llll}
\hline & Y & Q & R & Z \\
\hline Alt 1 & 0,24 & 0,51 & 0,55 & 0,85 \\
Alt 2 & 0,18 & 0,44 & 0,48 & 0,75 \\
Alt 3 & 0,16 & 0,4 & 0,44 & 0,72 \\
Alt 4 & 0,21 & 0,48 & 0,50 & 0,79 \\
\hline
\end{tabular}

Apresenta-se na figura 1 a representação aproximada dos fuzzy indexes obtidos, bem como das funções de pertinência dos conjuntos maximizante e minimizante, considerados como lineares $(\vartheta=1)$.

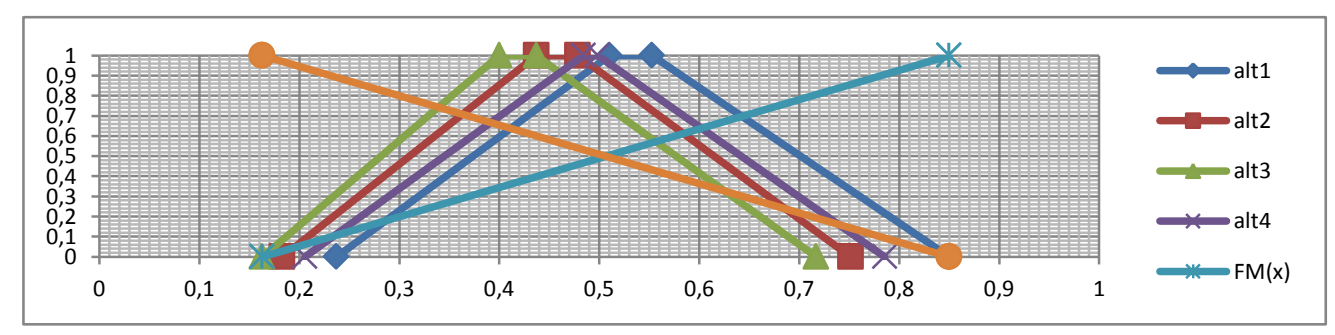

Figura 1 - Fuzzy Indexes 
As utilidades das alternativas, obtidas da aproximação apresentada na seção 2.2, são Alternativa 1 -0,53; Alternativa 2 - 0,45; Alternativa 3-0,41; e Alternativa $4-0,49$.

\subsection{INDICES BAAS \& KWAKERNAAK}

Utilizando a metodologia de (Baas and Kwakernaak 1977), obtemos os seguintes índices $R_{i}$ para as alternativas: $\bar{R}_{1}(0,65 ; 0,89 ; 1) ; \bar{R}_{2}(0,51 ; 0,78 ; 0,91) ; \bar{R}_{3}(0,45 ; 0,69 ; 0,92)$; e $\bar{R}_{1}(0,56 ; 0,82 ; 0,94)$.

O resultado aproximado em forma de números triangulares é apresentado na figura 2.

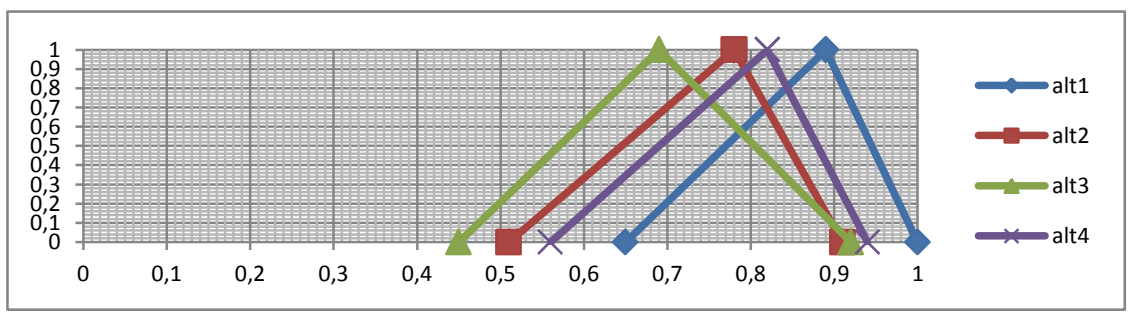

Figura 2- Índices R - Baas \& Kwakernaak

Cabe ressaltar que, apesar dos pesos e avaliações serem expressos em números fuzzy triangulares, o procedimento de obtenção dos índices não se utiliza das propriedades de multiplicação e divisão destes números. Uma explicação mais didática do procedimento pode ser encontrada em (Khamaran 2008). De posse dos índices torna-se possível efetuar os cálculos de $\mu_{I}(i)$ que representam a avaliação final da alternativa.

$\mu_{I}(1)=1 ; \mu_{I}(2)=0,61 ; \mu_{I}(3)=0,34 ;$ e $\mu_{I}(4)=0,81$. Relembra-se que para este cálculo é necessário o estabelecimento de todos os $\bar{R}_{i}$. Apresenta-se na figura 3 a plotagem de $P_{1}=$ $\left(R, \mu_{P 1}\right)$ que representa a extensão de quão melhor é a alternativa 1 sobre as demais.

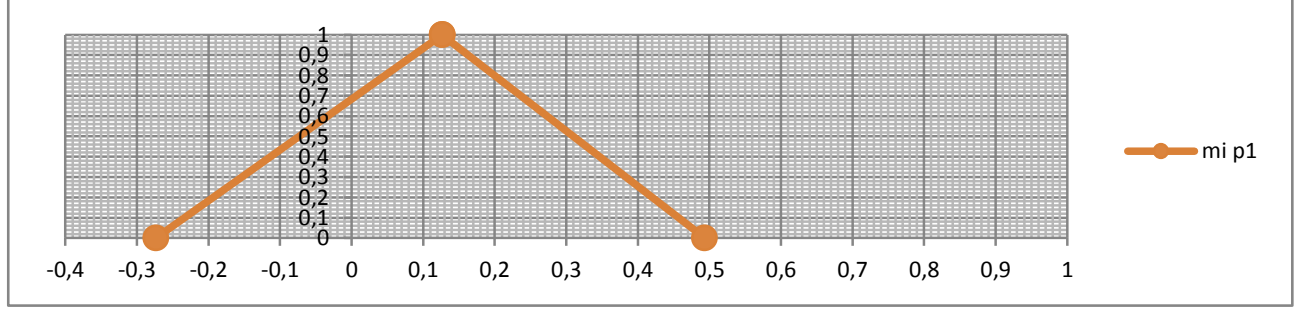

Figura 3 - Pi Alternativa 1

\subsection{MODELO COSENZA}

Conforme mencionado na seção 2.3 o modelo Coppe-Cosenza possibilita a avaliação de diversos projetos simultaneamente. Como há apena um projeto (o problema de uma facilidade locacional) a matriz $\tilde{A}$ apresenta apenas uma linha, como apresentado na tabela 6 :

Tabela 6 - Matriz de requisitos

\begin{tabular}{c|ccccccc}
\hline & $\mathbf{f}_{1}$ & $\mathbf{f}_{2}$ & $\mathbf{f}_{3}$ & $\mathbf{f}_{4}$ & $\mathbf{f}_{5}$ & $\mathbf{f}_{6}$ & $\mathbf{f}_{\mathbf{7}}$ \\
\hline$\widetilde{A_{1}}$ & Cond & Pouco Cond & Cond & Cond & Crítico & Pouco Cond & Pouco Cond \\
\hline
\end{tabular}

Enquanto a matriz de oferta é extraída da seção 3.1. Utilizando a matriz de cotejo que 
estabelece a correspondência entre requisitos e oferta das alternativas, efetuamos $C=A \otimes$ $B=\left(c_{i k}\right)_{m}$. Consolida-se o resultado final, normalizando-o pelo número de critérios. Obtendo-se os seguintes índices: Alternativa 1 - 1,08; Alternativa $2-0,77$; Alternativa 3 0,59 ; e Alternativa $4-0,86$.

\section{COMPARAÇÕES E CONCLUSÕES}

Obtemos para as três metodologias, considerando os critérios estabelecidos e as avaliações executadas, a mesma ordenação para as alternativas, apresentada na tabela 7 .

Tabela 7 - Classificação Alternativas

\begin{tabular}{l|llllll}
\hline Alternativa & \multicolumn{2}{l}{ Liang \& Wang } & \multicolumn{2}{l}{ Baas \& Kwakernaak } & \multicolumn{2}{l}{ Coppe-Cosenza } \\
\hline \multirow{3}{*}{1} & rank & Ut & rank & $\boldsymbol{\mu i}$ & rank & ind \\
\cline { 2 - 7 } 2 & primeiro & 0,53 & primeiro & 1 & primeiro & 1,08 \\
3 & terceiro & 0,45 & terceiro & 0,61 & terceiro & 0,77 \\
4 & quarto & 0,41 & quarto & 0,34 & quarto & 0,59 \\
& segundo & 0,49 & segundo & 0,81 & segundo & 0,86 \\
\hline
\end{tabular}

Observa-se que, apesar de haver a mesma ordem de precedência, a dominância relativa de cada opção é mostrada em escalas diferentes, podendo incorrer em interpretações diferenciadas na análise de sensibilidade (para verificação de alteração de avaliação a determinado critério, por exemplo).

A simplicidade de execução é bem diversa ao compararem-se os métodos. O modelo de Baas e Kwakernaak obriga a uma inspeção para construção da avaliação agregada de cada alternativa, crescendo exponencialmente com o número de critérios. (Para a construção de um alfa-cut zero em dois critérios para uma alternativa são geradas 16 possibilidades para exame). Sendo, portanto, menos recomendável para problemas com muitos critérios. Outras maneiras de agregação também podem ser encontradas em (Khamaran 2008).

Quanto à flexibilidade, o modelo Coppe-Cosenza (Cosenza et al. 2006) (Lima et al. 2009) possibilita utilização de outros algoritmos de avaliação. Este fato permite ajuste para uso do modelo em outros problemas, no qual pode ser alterado o efeito compensatório entre os critérios por meio da mudança do operador de agregação.

Torna-se importante, portanto, a escolha do algoritmo ao utilizar o modelo CoppeCosenza, cuja estrutura seja adequada à avaliação. Neste caso, optou-se pelo algoritmo apresentado na seção 2 para quer não houvesse o tratamento igual de penalidade (decorrente da inadequação a determinado critério) e da recompensa em atingir o critério de maneira melhor que o exigido.

Nota-se que a estrutura de agregação de diversos decisores está explícita somente em Liang \& Wang (Liang and Wang 1991). A utilização de consenso entre múltiplos decisores é utilizada em (Narasimhan 1979) (que menciona a adoção dos procedimentos de Baas \& Kwaakernaak), e também pode ser utilizado no modelo Coppe-Cosenza. Por outro lado, destaca-se que o modelo Coppe-Cosenza é capaz de incluir de novas alternativas paulatinamente, por não necessitar da explicitação de todas alternativas para a agregação de 
sua avaliação final. Este detalhe sutil pode ter grande impacto quando são apresentadas novas alternativas no decorrer do estudo de localização e para a análise de sensibilidade das decisões.

Considera-se que, além de estudos locacionais, os métodos poderiam ser utilizados em um amplo leque de problemas relacionados ao processo decisório na esfera militar. $\mathrm{O}$ modelo Liang-Wang, por exemplo, pode ser utilizado para avaliação de equipamentos, utilizando a estrutura para agregação de aspectos qualitativos e financeiros. Particularmente o modelo Coppe-Cosenza também pode utilizar diferentes avaliadores para cada critério, aspecto que também poderia ser útil em um estudo de Estado-Maior (EGN 2014a).

Finalmente, destaca-se outro aspecto relacionado à esfera militar, que diz respeito à impossibilidade de utilização dos métodos de Liang-Wang e de Baas-Kwaakernaak para análise de adequabilidade e de exequibilidade das alternativas. Ou seja, todas as alternativas nestes métodos devem ser consideradas exequíveis e adequadas (EGN 2014b), servindo como uma avaliação tão somente quanto à aceitabilidade. A utilização de fatores específicos no modelo Coppe-Cosenza possibilita, tal qual mencionado na seção 2, aspecto de avaliação similar a veto, ou eliminatório, o qual pode ser usado para este fim.

\section{REFERÊNCIAS BIBLIOGRÁFICAS}

Baas, SM, and H Kwakernaak. 1977. "Rating and Ranking of Multiple-Aspect Alternatives Using Fuzzy Sets.” Automatica 13: 47-58.

Brasil. 2008. Estratégia Nacional de Defesa. Decreto 6703 de 18 de dezembro de 2008.

Cosenza, Carlos Alberto Nunes. 2009. "Hierarchy Models for the Organization of Economic Spaces - Metrics and Operators for Facility Site Selection". Cambridge.

Cosenza, Carlos Alberto Nunes, César das Neves, Fernando Rodrigues Lima, and Flávio Raposo da Almeida. 2006. "Sistema de Informações Gráficas Georeferenciadas Para Estudos de Localização de Plantas de Biodiesel no Centro-Sul Brasileiro.” In XXVI Encontro Nacional de Engenharia de Produção, 1-8. Fortaleza: ABEPRO.

EGN. 2014a. "Estudo de Estado-Maior."

http://www.egn.mar.mil.br/arquivos/eem/eem/eem_1.htm.

2014b. "Estudo de Estado-Maior Capitulo 2."

http://www.egn.mar.mil.br/arquivos/eem/eem/eem_5.htm.

Khamaran, Cengiz, ed. 2008. FUZZY MULTI-CRITERIA DECISION MAKING Springer Optimization and Its Applications. Springer. 
Liang, Gin-Shu, and Mao-Jiun J. Wang. 1991. "A Fuzzy Multi-Criteria Decision-Making Method for Facility Site Selection.” The International Journal of Production 29 (11): 23132330. http://www.tandfonline.com/doi/abs/10.1080/00207549108948085.

Lima, Fernando Rodrigues, Carlos Alberto Nunes Cosenza, and César das Neves. 2009. "Modelos de Localização Em Engenharia Urbana." In Simpósio de Pós-Graduação Em Engenharia Urbana.

Narasimhan, R. 1979. “A FUZZY SUBSET CHARACTERIZATION OF A SITE$\square$ SELECTION PROBLEM.” Decision Sciences. http://onlinelibrary.wiley.com/doi/10.1111/j.1540-5915.1979.tb00051.x/abstract.

Nobre, R C M, O C Rotunno Filho, W J Mansur, M M M Nobre, and C A N Cosenza. 2007. "Groundwater Vulnerability and Risk Mapping Using GIS, Modeling and a Fuzzy Logic Tool." Journal of Contaminant Hydrology 94 (3-4) (December 7): 277-92. doi:10.1016/j.jconhyd.2007.07.008. http://www.ncbi.nlm.nih.gov/pubmed/17728007. 